\title{
ABO-incompatible kidney transplantation
}

Pre-emptive living kidney transplantation is the best choice of therapy to treat patients with advanced renal insufficiency. Unfortunately in up to one third of all cases kidney donation was refused due to blood group incompatibility. Limitations in donor availability for kidney transplantation therefore require that $\mathrm{ABO}$-incompatible transplantation is safely established. Solid organ transplantation between donors and recipients with incompatible blood groups, however, was contraindicated because of the high risk of hyperacute rejection.

Now the group of Georg Böhmig at the Medical University in Vienna describes their experience with ABOincompatible kidney transplantation and adds further evidence on the role $\mathrm{ABO}$-incompatible organ transplantation might play in the near future [1].

Historically, ABO-incompatible kidney transplantations have only been performed after several preoperative sessions of plasmapheresis to remove existing anti-A or anti-B antibodies. This was followed by splenectomy and a conventional triple-drug immunosuppressive protocol, reinforced with anti-lymphocyte globulin and B-cell-specific drugs, such as cyclophosphamide or deoxyspergualin, to prevent rebound of antibodies. However, because splenectomy, a reinforced immunosuppressive protocol, and multiple plasmapheresis sessions have been associated with significant morbidity as risk of infections and loss of physiological plasma components that include coagulation factors, hormones, and antiviral and antibacterial immunoglobulin G (IgG) and immunoglobulin M (IgM) and has been associated even with high mortality, most centers have refused to perform ABO-incompatible organ transplantations [2]. This has changed when a new protocol was introduced in Stockholm, Sweden, in 2001. Almost 200 ABO-incompatible transplantations have since been performed in more than 20 centers with this protocol in Europe [3]. Now the group of Georg Boehmig adds further evidence that this technique currently used is safe and can be offered to our patients [1].

As AB blood group antigens are expressed not only on red blood cells, but also on endothelial cells of arteries, glomerular/ peritubular capillaries and veins and also on distal tubular epithelium of the donor organ anti-A/B antibody induce (hyper-) acute humoral graft rejection [4]. Therefore, circulating anti-A/B antibodies

Correspondence: Prof. Dr. Friedrich Thaiss, Universitäts-Klinikum Hamburg Eppendorf UKE, Transplantations-Center \& III. Medizinische Klinik und Poliklinik, Martinistraße 52, 20246 Hamburg, Germany, E-mail: thaiss@uke.uni-hamburg.de need to be reduced in the recipient's serum prior to transplantation in order to overcome anti-A/B antibodymediated rejection and early graft loss in ABO-incompatible kidney transplantation. The most widely used techniques to reduce the amount of pre-existing anti-A or anti-B antibodies have been the plasmapheresis and double-filtration plasmapheresis most often used in Japan $[5,6]$. In theory, this technique should be able to remove both IgG and IgM molecules while allowing albumin to pass through. Attempts have been made to invent even more specific immunoadsorbing systems that adsorb only the IgG and IgM antibodies or, ideally, only the subclasses of IgG and IgM antibodies that are targeted, namely anti- A/B antibodies. Two such immunoadsorption columns are available at the moment: the protein A adsorption column (Immunosorba ${ }^{\circledR}$ ) that adsorbs immunoglobulins, and the ABO antigen-specific column (Glycosorb ${ }^{\circledR}$ ) that adsorbs the more specific anti-A or anti-B antibodies regardless of immunoglobulin class or subclass. The protein A column (Immunosor$\mathrm{ba}^{\circledR}$ ) contains protein A as a ligand, covalently immobilized to a sepharose matrix. The columns are used to remove antibodies and immune complexes from the patient's plasma and other proteins bound to immunoglobulins. Plasma is separated from whole blood by filtration or centrifugation and returned to the patient after being processed through the column. Because only the immunoglobulin antibodies are adsorbed, no volume replacement is necessary $[7,8]$. The Glycosorb ABO immunoadsorption columns have been developed by the Swedish biotech company Glycorex Transplantation $\mathrm{AB}$ and contains synthetic terminal trisaccharides from A or B ABO blood group antigen covalently bound to Sepharose particles. The Glycosorb-A column is used to deplete anti-A whereas the Glycosorb-B column is used to deplete anti-B. Plasma is separated from the whole blood and then processed through the column before being reinfused. The columns effectively deplete anti-A or anti-B antibodies. Because the antigen-specific immunoadsorption specifically depletes only anti-A or anti-B antibodies, side effects usually associated with therapeutic plasma exchange are absent. Thus, with these two columns there are no coagulation disorders, no nonspecific protein adsorption, no activation of coagulation factors, and no effect on the antibody titers against previously encountered antigens such as viruses and vaccinations. Until now more than 2000 immune adsorption procedures with these two techniques were performed worldwide in different centres without serious side effects.

Exact measurement of anti-blood groups $\mathrm{A}$ and $\mathrm{B}$ antibody titers is critical for successful ABO-incompati- 
ble living kidney transplantation. However, no standard method for quantitative determination has yet been established. In most institutions performing ABO-incompatible transplantations, the indirect antiglobulin test is used to determine the initial IgG anti-A or anti-B titer against donor erythrocytes. However, there seem to be many detailed technical differences and large interinstitutional variations in the titration of anti-ABO antibodies. When four different techniques were compared, the Tube Technique, BioVue Column Agglutination Technology, DiaMed-ID Micro Typing System, and flow cytometry (FCM), only the FCM technique yielded a very consistent outcome in repeated measurements [9, 10]. The titer level at which a renal transplantation can safely be performed has not unequivocally been established not at least due to methodical problems as mentioned above. Most authors, however, aim at titer levels of < 16 in "major" (Al and B) incompatible cases. According to preoperative titers immunoadsorptions are performed on days $-6,-5,-2$, and -1 and two plasma volumes are processed at each session. Posttransplantation, one plasma volume was processed pre-emptively on days $+2,+5$, and +8 , however the necessity of the preemptive adsorption procedures after transplantation has been questioned recently. Some patients needed more immunoadsorptions to reach a titer of $1: 8$ or less before transplantation.

Having significantly lowered the AB-antibodies the kidney transplantation protocol in most centers utilizes a standard tacrolimus/ mycophenolate mofetil/ prednisolone-based immunosuppressive protocol and the monoclonal humanized anti-CD20 antibody rituximab is used as induction therapy given once 4 weeks before transplantation $[1,3]$.

After transplantation acute antibody-mediated rejection (AAMR) is known to be the primary cause of early graft loss in ABO-incompatible kidney transplantation, some studies reported that AAMR still occurred in up to $30 \%$, however, most studies did not examine the role of additional anti-HLA antibodies in these patients. Early protocol kidney biopsies in these patients are therefore necessary to demonstrate humoral rejection $[11,12]$. In biopsies of initially HLA-incompatible renal allografts a strong association between proximal tubular cell C4d staining and neutrophil margination has previously been described [13, 14]. By contrast, in protocol biopsies of ABO-incompatible allografts with stable function proximal tubular cell C4d staining was positive in $80 \%$, as also shown by Boehmig et al in the present paper [1]. These findings therefore suggest that in biopsies of $\mathrm{ABO}$-incompatible grafts that are performed for early dysfunction, proximal tubular cell C4d may be associated with antibody mediated acute rejection and graft injury, whereas in stably functioning grafts, proximal tubular cell C4d staining is frequently not associated with antibody mediated rejection and may reflect graft accommodation [15]. In contrast, in HLA antibody incompatible transplantation, C4d deposition was highly associated with other histologic abnormalities characteristic of acute humoral rejection such as thrombotic microangiopathy and peritubular capillary neu- trophil margination. Thus, although peritubular capillary C4d deposition is considered to be an important histologic indicator of antibody-endothelial cell interaction, and is a key finding in the diagnosis of acute humoral rejection, the detection of $\mathrm{C} 4 \mathrm{~d}$ deposition in ABO-incompatible kidney transplants with stable kidney function should not be considered to indicate active acute humoral rejection $[15,16]$.

Graft function is comparable with that of ABOcompatible kidney transplantation also in a longer-term perspective up to five years after transplantation [17]. The overall event-free time, defined as time free of death, graft loss, or rejection, was also equivalent to ABO-compatible kidney transplantation. Nor could any significant difference in the incidence of acute rejection complications be observed. Infectious complications have been reported after the use of rituximab for other indications, but have not been well studied in the solid organ transplant population. The data available up to now suggest that there is a non significant trend toward an increased rate of infectious complications with rituximab therapy in ABO-incompatible kidney transplant recipients. Thus overall ABO-incompatible kidney transplantation using immunoadsorption procedures and rituximab in combination with triple immunosuppressive therapy is equivalent to standard $\mathrm{ABO}$-compatible living donor kidney transplantation. ABO-incompatible kidney transplantation using the current protocol is also cost saving when compared with continuing dialysis for wait listed transplantation candidates [18, 19].

Although ABO-incompatible kidney transplantation is safely established in different transplantation centers in Europe as also demonstrated by the Vienna experience published in this issue of WKW there are many issues which need to be addressed in the near future. What we definitely need is long term follow up of those patients which have already been transplanted with the new protocols when compared with ABO-compatible kidney transplantation candidates. What we also urgently need is standardization of the blood group antibody titer determination protocols currently used. This will help to further define the specificity of the immunoadsorption columns used and redefine the routine use of immunoglobuline before transplantation as currently performed in most centers. We also need short and long term follow up protocol biopsies in these patients to better understand the factors important for induction of accommodation, a state that may support longterm survival of vascularized organs, versus humoral rejection. This will help to further define the modality of rituximab application in these patients. What protocol and doses of rituximab should we use; is counting of peripheral B-cells specific enough or do we need more widespread use of B-cell counting in the kidney or even lymph node biopsies? New monoclonal B-cell antibodies will further help to define the functional role Bcells play in ABO-compatible and-incompatible kidney transplantation [20-22]. Also HLA-antibodies have to be thoroughly determined in the follow up of these patients as an increasing number of the ABO-incompatible 
kidney transplantations are performed in patients which have had an ABO-compatible organ transplantation before. We also need to define rules which patients should be offered an ABO-incompatible kidney transplantation or which patients should be better included in a living donor list exchange program, which is not available in all European countries.

In conclusion, ABO-incompatible living kidney transplantation can be offered to our patients with advanced kidney disease as a safe procedure. The group of Georg Boehmig at the University Hospital in Vienna now adds further evidence to the procedure currently used in ABO-incompatible kidney transplantation. To get more insight into the role ABO-incompatible organ transplantation might play in the near future transplantation centers currently involved in these processes should share their data to answer the unresolved issues we are concerning.

\section{Friedrich Thaiss}

\section{References}

1. Haidinger M, Schmaldienst S, Körmöczi G, et al (2009) Vienna experience of ABO-incompatible living donor kidney transplantation. Wien Klin Wochenschr 121: 247-255

2. Gloor J, Stegall MD (2007) ABO incompatible kidney transplantation. Curr Opin Nephrol Hypertens 16: 529534

3. Tyden G, Donauer J, Wadström J, et al (2007) Implementation of a protocol for ABO-incompatible kidney transplantation - a three-center experience with 60 consecutive transplantations. Transplantation 83: 1153-1155

4. Breimer ME, Mölne J, Norden G, et al (2006) Blood group A and B Antigen Expression in human kidneys correlated to A1/A2/B, Lewis, and secretor status. Transplantation 82: 479-485

5. Tobian AA, Shirey RS, Montgomery RA, Ness PM, King KE (2008) The critical role of plasmapheresis in ABO-incompatible renal transplantation. Transfusion 48: 2453-2460

6. Derfler K, Druml W (2004) Die Immunadsorption (IAS) wird „erwachsen“. Wien Klin Wochenschr 116: 699-702

7. Tyden G, Kumlien G, Efvergren M (2007) Present techniques for antibody removal. Transplantation 84: S7-S29

8. Kumlien G, Ullström L, Losvall A, et al (2006) Clinical experience with a new apheresis filter that specifically depletes ABO blood group antibodies. Transfusion 46: 1568-1575
9. Rydberg L, Skogsberg U, Mölne J (2007) ABO antigen expression in graft tissue: is titration against donor erythrocytes relevant? Transplantation 84: S10-S12

10. Yung G, Valli PV, Starke A, et al (2007) Flow cytometric measurement of $\mathrm{ABO}$ antibodies in $\mathrm{ABO}$-incompatible living donor kidney transplantation. Transplantation 84: S20-S23

11. Racusen LC, Haas M (2006) Antibody-mediated rejection in renal allografts: lessons from pathology. Clin J Am Soc Nephrol 1: 415-420

12. Setoguchi K, Ishida H, Shimmura H, et al (2008) Analysis of renal transplant protocol biopsies in $\mathrm{ABO}$-incompatible kidney transplantation. Am J Transplant 8: 86-94

13. Böhmig G (2006) Alloantibodies-mediated kidney transplant rejection: a pair of continuing approaches, and with nonetheless many open questions. Wien Klin Wochenschr 118: 373-381

14. Feucht HE (2006) Die Entdeckung des kapillären C4d in Nierentransplantaten und die "Renaissance“ der humoralen Abstoßung. Wien Klin Wochenschr 118: 426-434

15. Haas M, Segev DL, Racusen LC, Bagnasco SM, Locke JE, Warren DS, Simpkins CE, Lepley D, King KE, Kraus ES, Montgomery RA (2009) C4d deposition without rejection correlates with reduced early scarring in ABO-incompatible renal allografts. J Am Soc Nephrol 20: 197-204

16. Toki D, Ishida H, Setoguchi K, Shimizu T, Omoto K, Shirakawa $\mathrm{H}$, Iida S, Horita S, Furusawa M, Ishizuka T, Yamaguchi Y, Tanabe K (2009) Acute antibody-mediated rejection in living ABO-incompatible kidney transplantation: longterm impact and risk factors. Am J Transplant 9: 567-577

17. Genberg H, Kumlien G, Wennberg L, Tyden G (2007) Longterm results of $\mathrm{ABO}$-incompatible kidney transplantation with antigen-specific immunoadsorption and rituximab. Transplantation 84: S44-S47

18. Schwartz J, Stegall MD, Kremers WK, Gloor J (2006) Complications, resource utilization, and cost of ABO-incompatible living donor kidney transplantation. Transplantation 82: 155-163

19. Schnitzler M, Machnicki G (2006) ABO-incompatible living donor transplantation: is it economically "compatible"? Transplantation 82: 168-169

20. Steinmetz OM, Lange-Hüsken F, Turner JE, et al (2007) Rituximab removes intrarenal B cell clusters in patients with renal vascular allograft rejection. Transplantation 15: 842-850

21. Segerer S, Schlöndorff D (2008) B cells and tertiary lymphoid organs in renal inflammation. Kidney Int 73: 533537

22. Dörner T, Burmester GR (2008) New approaches of B-celldirected therapy: beyond rituximab. Curr Opin Rheumatol 20: 263-268 